began to materialise in 2002 when the Global Fund to Fight AIDS, Tuberculosis and Malaria was established. The scale-up of malaria interventions, especially for vector control, began in earnest in 2004 in sub-Saharan Africa but by 2007 only less than $20 \%$ of children in Africa had slept under an insecticidetreated net. In addition, it is only from 2006 that the widespread use of artemesinin combination therapy (ACT) started to occur on a wide scale. By 2015, however, substantial coverage with both vector and treatment interventions had been achieved. Consequently, the malaria burden has decreased across sub-Saharan Africa. Here I review the current malaria situation in sub-Saharan Africa and discuss opportunities and challenges. I discuss some of the analytical work, including mapping that contributed to our understanding of the epidemiology of malaria and progress during the RBM era. I present examples of how this research has led to changes in policy and practice globally and in sub-Saharan Africa. I discuss the current major research needs and policy gaps in the malaria elimination agenda and how these may be applicable to other diseases in the EDCTP remit. I conclude with suggestions on the potential role of EDCTP in supporting clinical trials and capacity strengthening towards malaria elimination.

\section{PS-006 MALARIA}

Abdisalan Noor. WHO, Switzerland

10.1136/bmjgh-2016-000260.6

At the beginning of the Roll Back Malaria (RBM) initiative around 2000, sub-Saharan Africa was going through a major malaria epidemic, compounded by the failure of chloroquine and emerging resistance to sulphadoxine-pyremithamine, as the first-line treatments for uncomplicated malaria. The initial call for increased funding through the 2000 Abuja Declaration only 